\title{
Unique features of apicoplast DNA gyrases from Toxoplasma gondii and Plasmodium falciparum
}

\author{
Soshichiro Nagano', Ting-Yu Lin', Jyotheeswara Reddy Edula ${ }^{1,2}$ and Jonathan Gardiner Heddle ${ }^{1 *}$
}

\begin{abstract}
Background: DNA gyrase, an enzyme once thought to be unique to bacteria, is also found in some eukaryotic plastids including the apicoplast of Apicomplexa such as Plasmodium falciparum and Toxoplasma gondii which are important disease-causing organisms. DNA gyrase is an excellent target for antibacterial drugs, yet such antibacterials seem ineffective against Apicomplexa. Characterisation of the apicoplast gyrases would be a useful step towards understanding why this should be so. While purification of active apicoplast gyrase has proved impossible to date, in silico analyses have allowed us to discover differences in the apicoplast proteins. The resulting predicted structural and functional differences will be a first step towards development of apicoplast-gyrase specific inhibitors.

Results: We have carried out sequence analysis and structural predictions of the enzymes from the two species and find that $P$. falciparum gyrase lacks a GyrA box, but T. gondii may retain one. All proteins contained signal/transport peptides for localization to the apicoplast but T. gondii Gyrase B protein lacks the expected hydrophobic region. The most significant difference is in the GyrA C-terminal domain: While the cores of the proteins, including DNA binding and cleavage regions are essentially unchanged, both apicoplast gyrase A proteins have C-terminal domains that are significantly larger than bacterial counterparts and are predicted to have different structures.

Conclusion: The apicoplast gyrases differ significantly from bacterial gyrases while retaining similar core domains. $T$. gondii Gyrase B may have an unusual or inefficient mechanism of localisation to the apicoplast. P.falciparum gyrase, lacks a GyrA box and is therefore likely to be inefficient in DNA supercoiling. The C-terminal domains of both apicoplast Gyrase A proteins diverge significantly from the bacterial proteins. We predict that an additional structural element is present in the C-terminal domain of both apicoplast Gyrase A proteins, including the possibility of a $\beta$-pinwheel with a non-canonical number of blades. These differences undoubtedly will affect the DNA supercoiling mechanism and have perhaps evolved to compensate for the lack of Topoisomerase IV in the apicoplast. These data will be useful first step towards further characterisation and development of inhibitors for apicoplast gyrases.
\end{abstract}

Keywords: Topoisomerase, DNA gyrase, malaria toxoplasmosis, Negative supercoiling, Apicoplast

\section{Background}

Apicomplexa are a group of unicellular protist parasites, most of which contain a plastid known as the apicoplast. Apicoplasts are the result of secondary endosymbiosis in which a prokaryotic cyanobacterium was incorporated into a unicellular eukaryote which was subsequently engulfed by a second eukaryote $[1,2]$. As a result of this process, the apicoplast contains the remnants of the bacterial plasmid and has four membranes, which from

\footnotetext{
* Correspondence: heddle@riken.jp

'Heddle Initiative Research Unit, RIKEN, 2-1 Hirosawa, Wako, Saitama 351-0198, Japan

Full list of author information is available at the end of the article
}

outside inward are the second host's endomembrane, the first host's plasma membrane and the two apicoplast membranes [2].

The Apicomplexa are of interest because they count amongst their number several important human pathogens. Foremost amongst these are Plasmodium species, responsible for malaria, a disease which in 2010 infected approximately 200 million people resulting in over 600,000 deaths [3] and Toxoplasma gondii which can cause dangerous complications in the immune-compromised, is classified by the CDC as a "neglected parasitic disease" and is the biggest cause of death from foodborne illness in the USA [4].

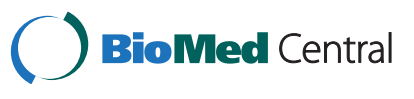

(c) 2014 Nagano et al.; licensee BioMed Central. This is an Open Access article distributed under the terms of the Creative Commons Attribution License (http://creativecommons.org/licenses/by/4.0), which permits unrestricted use, distribution, and reproduction in any medium, provided the original work is properly credited. The Creative Commons Public Domain Dedication waiver (http://creativecommons.org/publicdomain/zero/1.0/) applies to the data made available in this article, unless otherwise stated. 
Treatments for both toxoplasmosis (caused by T. gondii) and malaria exist but are not without their drawbacks. Most notably in the case of malaria, resistance to existing treatments is widespread [5] and has recently come to include artemisinin, the major component of the most effective current treatment [6,7]. Progress has been made in development of a malaria vaccine but results of large scale clinical trials have been disappointing [8] although most recent small scale trials do show promise [9]. Drug resistance in $T$. gondii is also regarded as problematic [10].

Apicoplasts are known to be essential for the survival of apicomplexan cells due to their numerous roles (reviewed by van Dooren and Striepen [2]) these include synthesis of heme, iron-sulfur clusters, fatty acids and isoprenoids. The requirement for an apicoplast was initially demonstrated in $T$. gondii, which was unable to survive when apicoplast DNA replication was inhibited [11], or when the apicoplast was absent [12]. In Plasmodium their essential function in the blood stages appears to be synthesis of isoprenoid precursors [13].

The indispensability of the apicoplast together with fact that it is a eubacteria-derived plastid raises the possibility of exploiting it for specific targeting of pathogenic Apicomplexa with antibacterial drugs without affecting the human host [14].

One of the most successful antibacterial drug targets to date has been DNA gyrase ("gyrase"), a type II topoisomerase that has the unique ability to carry out ATPdependent negative supercoiling of DNA [15]. Gyrase consists of GyrA and GyrB proteins with the functional enzyme being an $\mathrm{A}_{2} \mathrm{~B}_{2}$ heterotetramer. The supercoiling mechanism [16] is complex and includes a step in which the enzyme produces a transient double-stranded break in the substrate DNA (see Figure 1). GyrA consists of an Nterminal domain which contains a DNA binding region and the active site tyrosine, involved in DNA cleavage via formation of a phosphotyrosine bond and a C-terminal domain (CTD) that wraps DNA with the appropriate handedness for negative supercoil generation and delivers it to GyrB. The CTD structure responsible for this wrapping is termed a $\beta$-pinwheel fold [17]. This is similar to the $\beta$-propeller fold [18] overall but with different topology in its repeating units ("blades"). GyrB consists of an $\mathrm{N}$-terminal domain containing the region responsible for ATP binding and hydrolysis and the transducer region which connects the ATPase domain to the TOPRIM (topoisomerase-primase [19]) domain. The C-terminal region of GyrB contains the TOPRIM domain involved in the DNA cleavage reaction (Figure 1).

Gyrase poisons work by inhibiting the religation of cleaved DNA, leading to fragmentation and cell death. The highly successful fluoroquinolone class of antibacterials function in this way and also target topoisomerase IV ("topo IV"), a closely related bacterial type II topoisomerase, in the same fashion. Topo IV itself is structurally highly similar to DNA gyrase, also functioning as a heterotetramer $\left(\operatorname{ParC}_{2}, \operatorname{ParE}_{2}\right)$. Like gyrase it requires ATP hydrolysis to carry out its function, which is mainly decatenation of daughter chromosomes rather than negative supercoiling. Differences in the $\mathrm{C}$ terminal domain of GyrA/ParC subunits appear to account for this difference in function [22].

Until recently, gyrase was thought to occur only in bacterial cells. It is now known that, while apparently not occurring in humans and most other higher eukaryotes, it is found in plant plastids [23]. A malarial gyrase, whose existence had long been suggested by evidence such as the activity of fluoroquinolones against Plasmodium and $T$. gondii [11,24-27], was finally shown to exist through sequencing of the P. falciparum genome [28] and is localized to the apicoplast. Apicoplast gyrase has been mooted as a potentially useful therapeutic target $[14,29]$ although fluoroquinolone activity in vitro culture is usually much higher than in an infected host where results are variable. In the case of in vitro experiments with $T$. gondii for example, ciprofloxacin (CFX) has an $\mathrm{IC}_{50}$ of $27.9 \mu \mathrm{g} / \mathrm{ml}[30]$ and in the case of $P$. falciparum in in vitro culture, initial results suggested an $\mathrm{IC}_{50}$ for CFX of $1.7 \mu \mathrm{g} / \mathrm{ml}$ for 24-hour experiments [26]. Mahmoudi et al. carried out a comprehensive in vitro test of 25 quinolones and fluoroquinolones against blood stages of P. falciparum [31] and found an $\mathrm{IC}_{50}$ for CFX of approximately $9.2 \mu \mathrm{g} / \mathrm{ml}$ and $3.4 \mu \mathrm{g} / \mathrm{ml}$ against chloroquine sensitive and resistant strains respectively. These numbers compare to $\mathrm{IC}_{50} \mathrm{~s}$ for $\mathrm{CFX}$ against $E$. coli in the approximate range of $0.011-0.015 \mu \mathrm{g} / \mathrm{ml}$ [32].

Interestingly, the way in which CFX affects $T$. gondii appears different to the effect on $P$. falciparum as $T$. gondii shows a clear "delayed death" where extending treatment time lowers the $\mathrm{IC}_{50}$, something which appears not to occur in P. falciparum for CFX [33] although it is seen for some other molecules.

There is much less information regarding the effectiveness of these drugs against the pathogens in an infected host ("in vivo"). CFX, while active against T. gondii in in vitro culture, apparently shows little effect against the parasite in vivo [27]. In contrast, the fluoroquinolone trovafloxacin is unusual in that it does show in vivo activity [27]. Poor in vivo performance in general could be related to complications caused by parasite life cycle or simply the additional physical barriers in place in the host cell. Differences could also be due to differences in structure or mechanism of action of the enzymes themselves. Mature T. gondii gyrase (Tg-gyrase) is $142 \%$ the size of E. coli gyrase (Ec-gyrase) in terms of number of amino acids and $P$. falciparum gyrase (Pf-gyrase) is $116 \%$, giving much scope for potential deviation from the $E$. coli "norm". To understand the poor effectiveness of gyrase- 


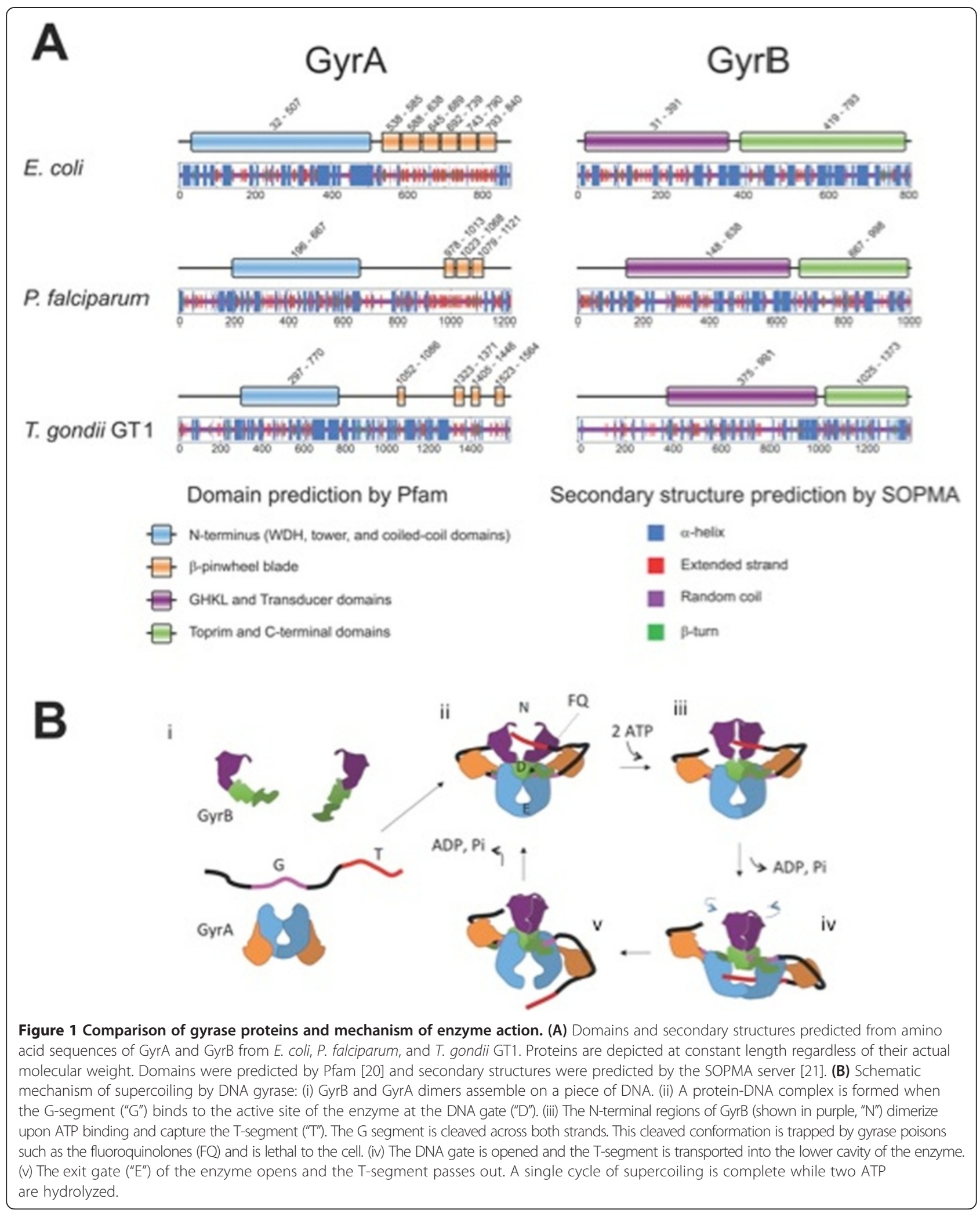

poisoning antibacterials against Apicomplexa it is very important to biochemically and biophysically characterize purified Pf- and Tg-gyrases in vitro.
Despite problems in expression and purification of Pfgyrase proteins, some biochemical experimental work has been carried out utilising various domains (summarised in 
Table 1): Full length Pf-GyrB has been characterized for ATPase activity which was found to be DNA-stimulated $[34,35]$ and shows a $K_{c a t}$ similar to that reported for the $43 \mathrm{kDa}$ domain of Ec-GyrB [34,36]. An N-terminal region of Pf-GyrB (residues 121-497, equivalent to the Ec-GyrB $43 \mathrm{kDa}$ ATPase domain) has also been produced and characterized and shows ATP hydrolysis activity at a somewhat lower rate than its E. coli counterpart [35].

Pf-GyrB also shows ATP-hydrolysis and DNA supercoiling activities when complemented with Ec-GyrA [35]. Furthermore the enzyme appears resistant to drugs that function by disrupting the ATP-binding site with the Ki value of coumermycin reported to be 500 times that of the E. coli protein [35] and the Ki value of novobiocin being approximately 3.5 times higher for Pf-GyrB compared to Ec-GyrB [34].

While full-length Pf-GyrA (residues 156-1222) has not been successfully expressed and purified, it has proved possible to produce the N-terminal DNA cleavagereunion domain (residues 163-540) [35]. This domain shows $\mathrm{CaCl}_{2}$-induced and CFX-induced DNA cleavage when combined with Pf-GyrB [35]. The expression and characterization of the full-length CTD of Pf-GyrA has not been reported to date, although an $\mathrm{N}$-terminal portion of it (residues 723-887) has been produced [35].

The lack of a purified full length Pf-GyrA has undoubtedly hampered progress in understanding Pf-gyrase. Still less is known of the Tg-gyrase proteins, the purification of which has not been reported to date (as is the case for all other apicomplexan gyrases). If these enzymes could be purified and characterized biochemically and structurally in comparison with standard eubacterial gyrase the data generated could help in the design of new inhibitors and may also point to a better understanding of the mechanism of action of gyrase itself.
In terms of structure, alignments for $P$. falciparum have suggested a close match between the $43 \mathrm{kDa} \mathrm{N}$ terminal GyrB domain of Ec-gyrase containing the ATP-binding and hydrolysis site and the equivalent region of Pf-GyrB and between the $59 \mathrm{kDa} \mathrm{N}$-terminal cleavage-reunion domain of Ec-GyrA and equivalent region of Pf-GyrA [34]. However, to the best of our knowledge such alignments have not been carried out for the C-terminal regions of Pf-GyrA and GyrB which is where the majority of sequence divergence is located. Tg-gyrase proteins appear never to have been subject to structural prediction studies.

Until purified holoenzymes become available, in silico approaches may be useful. Here we have used sequence analysis and structural alignments/predictions to compare DNA gyrase from $P$. falciparum and $T$. gondii to known bacterial enzymes (Table 2). Ultimate verification will of course require biochemical experiments but we hope that these initial results will spur further investigations into these interesting gyrases.

We show that the apicomplexan gyrases, as well as the obvious differences in size, contain signal and translocation peptides, which differ according to protein and species. Pf-gyrases contain numerous asparagines including repeat sequences. Perhaps the most significant differences between the proteins are found at the GyrA CTD, which varies significantly in size compared to the bacterial counterparts from which they show considerable divergence. We speculate that this divergence may include a DNA wrapping $\beta$-pinwheel domain with a different size/number of blades than has been seen to date in all known gyrases and/or an additional domain of unknown function. These differences may be related to modulation of supercoiling control in apicoplasts, which are not known to contain topoisomerase IV.

Table 1 Summary of experiments previously carried out with $P$. falciparum gyrase proteins

\begin{tabular}{|c|c|c|c|c|}
\hline Pf protein & Partner protein & Test & Result & References \\
\hline \multirow[t]{4}{*}{$\begin{array}{l}\text { GyrB (various } \\
\text { constructs) }\end{array}$} & & ATPase & $\begin{array}{l}\text { Decreased ATP turnover rate compared to } \\
\text { E. coli protein ATPase activity can be enhance }\end{array}$ & $\begin{array}{l}\text { Dar et al., } 2007 \text { [35]; Ram et al., } \\
2007 \text { [34]; Dar et al., } 2009 \text { [37] }\end{array}$ \\
\hline & & DNA binding & $\begin{array}{l}\text { DNA binding activity through } 45 \text { amino acid } \\
\text { insert region }\end{array}$ & Dar et al., 2009 [37] \\
\hline & & $\begin{array}{l}\text { Conformational change in } \\
\text { response to nucleotide }\end{array}$ & $\begin{array}{l}\text { Clamp closure can be modulated by } \\
\text { nucleotide binding }\end{array}$ & Dar et al., 2009 [37] \\
\hline & & Dimerization & Dimerization occurred in the absence of ATP & Dar et al., 2007 [35] \\
\hline \multirow[t]{3}{*}{ GyrB } & Ec-GyrA (full length) & ATPase & Enhanced ATP turnover rate & Dar et al., 2007 [35] \\
\hline & & Supercoiling & $\begin{array}{l}\text { Lower supercoiling activity compared to } \\
\text { E. coli gyrase }\end{array}$ & $\begin{array}{l}\text { Dar et al., } 2007 \text { [35]; Dar et al., } \\
2009 \text { [37] }\end{array}$ \\
\hline & & DNA cleavage & Introduced DNA breaks & $\begin{array}{l}\text { Dar et al., } 2007 \text { [35]; Dar et al., } \\
2009 \text { [37] }\end{array}$ \\
\hline \multirow[t]{2}{*}{ GyrB } & Pf-GyrA NTD & DNA cleavage & Introduced DNA breaks & Dar et al., 2007 [35] \\
\hline & & Supercoiling & Failed in DNA supercoiling & Dar et al., 2007 [35] \\
\hline GyrA NTD & Ec-GyrB (full length) & DNA cleavage & Introduced DNA breaks & Dar et al., 2007 [35] \\
\hline
\end{tabular}


Table 2 Summary of the protein sequences used in this study

\begin{tabular}{|c|c|c|c|}
\hline \multicolumn{4}{|l|}{ GyrA } \\
\hline Organism & Protein length & Molecular weight (Da) & Accession number \\
\hline E. coli & 875 & 96977.3 & [GenBank: ACl81094] \\
\hline M. tuberculosis & 838 & 92345.1 & [GenBank: AFR90340] \\
\hline S. aureus & 889 & 99624.9 & [GenBank: NP_373244] \\
\hline S. enterica & 878 & 97020.9 & [GenBank: WP_001281283] \\
\hline X. campestris & 899 & 99027.3 & [GenBank: WP_010366603] \\
\hline P. cynomolgi & 1266 & 141638.6 & [GenBank: P_004225009] \\
\hline P. falciparum & 1222 & 143144.1 & [GenBank: AAN36310] \\
\hline P. knowlesi & 1173 & 133858.5 & [GenBank: XP_002262302] \\
\hline P. vivax & 1263 & 144004.5 & [GenBank: EDL47594] \\
\hline T. gondii GT1 & 1594 & 175836.3 & [GenBank: EPR58555] \\
\hline T. gondii ME49 & 1278 & 142457.1 & [GenBank: XP_002369962] \\
\hline E. coli (ParC) & 752 & 83831.0 & [GenBank: NP_289596] \\
\hline \multicolumn{4}{|l|}{ GyrB } \\
\hline Organism & Protein length & Molecular weight (Da) & Accession number \\
\hline E. coli & 804 & 89866.6 & [GenBank: BAA20341] \\
\hline M. tuberculosis & 686 & 75323.9 & [GenBank: NP_334414] \\
\hline S. aureus & 644 & 72523.7 & [GenBank: NP_373243 ] \\
\hline S. enterica & 826 & 92159.9 & [GenBank: WP_006542968] \\
\hline X. campestris & 814 & 89688.5 & [GenBank: WP_010376402] \\
\hline P. cynomolgi & 908 & 102112.0 & [GenBank: XP_004225170] \\
\hline P. falciparum & 1006 & 116106.2 & [GenBank: AAN36469] \\
\hline P. knowlesi & 992 & 112346.0 & [GenBank: XP_002262476] \\
\hline P. vivax & 936 & 104905.8 & [GenBank: EDL44204] \\
\hline T. gondii GT1 & 1386 & 148239.4 & [GenBank: EPR58339] \\
\hline T. gondii ME49 & 1257 & 134533.3 & [GenBank: XP_002371198 ] \\
\hline E. coli (ParE) & 630 & 70243.7 & [GenBank: NP_417502] \\
\hline
\end{tabular}

Accession numbers were retrieved from NCBI (http://www.ncbi.nlm.nih.gov/).

\section{Results and discussion Sequence comparisons Sequence analyses of GyrA}

Bioinformatics analyses were carried out in order to gain deeper insights into the unique properties of Pf- and Tggyrases. Sequence alignments for Tg- and Pf- GyrAs and GyrBs all aligned best with topo IV structures. This likely reflects the lack of structural data for the relevant regions of gyrase proteins (i.e. full length GyrB and GyrA including the C-terminal DNA wrapping domains) rather than an indication that the structures actually more closely resemble $E$. coli topo IV.

Amino acid sequences of diverse gyrases were analysed using the MEGA analysis software [38]. Examples of several bacterial GyrAs and GyrBs were compared to examples from Plasmodium species along with sequences from $T$. gondii and E. coli topoisomerase IV. Two analyses were carried out. Firstly pairwise distances between sequences were calculated (Additional file 1: Tables S1 and S2). These showed that for gyrases, the differences in amino acid sequences were greatest between the bacterial gyrases and the Plasmodium gyrases for both GyrA and GyrB. Topoisomerase IV, as would be expected, was also the most different in the case of ParE compared to GyrBs, but in fact less different than the Plasmodium GyrA proteins in the case of ParC. Tg-GyrA fell between bacterial and Plasmodium sequences in terms of difference and Tg-GyrB was similar to Plasmodium proteins. The maximum parsimony bootstrap consensus trees for the proteins show a similar result with two clear clusters, one for bacterial proteins and another for Plasmodium proteins with topoisomerase IV and T. gondii gyrases being somewhat variable outliers (See Additional file 1: Figure S1). Sequences were also aligned with ClustalW $2.1[39,40]$ which showed that $25.6 \%$ of residues were identical between Ec-GyrA and Pf-GyrA. The value 
changes when analyses are delimited to individual domains identified by the Pfam server. Percentages of identical residues for the N-terminal domain (Ec-GyrA 32-507 encompassing WHD, tower, and coiled-coil domains) and the C-terminal domain (Ec-GyrA 538-840 encompassing the $\beta$-pinwheel domain) were $34.1 \%$ and $18.0 \%$, respectively. These observations are rationalized by lower homology of the C-terminal domain of Pf-GyrA with respect to Ec-GyrA. Analysis of the amino acid sequences of Ec-GyrA using the Pfam domain prediction server results in six $\beta$-sheet blades of the $\beta$-pinwheel motif being predicted in accordance with reported experimental results [41]. In contrast, only three such motifs are predicted from the Pf-GyrA sequence with the E-value set to 10 , the maximum value. This prediction is consistent with secondary structures predicted by the SOPMA structure prediction server [21], where $\beta$-sheets are predicted for those regions of Pf-GyrA (Figure 1A). At face value, this suggests the presence of a small $\beta$-pinwheel domain consisting of a cluster of no more than three blades (Figure 1, Additional file 1: Figure S2). All known gyrases have 6 blades [42]. Given that individual blades in Pf-GyrA appear to be approximately the same size as those found in other gyrases, it is difficult to envisage three blades alone to wrap DNA with a sufficiently acute angle to deliver it to the DNA clamp. Two possible explanations are that $i$ ) Pf-GyrA may be an example of a topo II with fewer than six $\beta$-pinwheel motifs (as is seen for E. coli topo IV [42]). ii) Pf-GyrA does in fact contain a $\beta$-pinwheel with a larger number of blades but they are of a sequence highly divergent from known structures and are thus not recognised by Pfam. For Tg-GyrA, the percentage of identical residues to Ec-GyrA is $33.5 \%$. In terms of the distribution of identical residues, similarity can be observed to that seen between Ec-GyrA and Pf-GyrA, i.e. a higher identity is found in the $\mathrm{N}$-terminal domain (41.5\%) compared to the C-terminal domain (26.7\%).

The N-terminal $59 \mathrm{kDa}$ fragment of Ec-GyrA bears a domain similar to the DNA-binding domain of the catabolite-activator protein (CAP) containing a helix-turnhelix $(\mathrm{HTH})$ motif and the tower domain [43]. A hallmark of topoisomerase, the active site tyrosine that forms a transient phosphodiester bond with the end of the Gsegment DNA is found in the HTH motif, which is part of the positively charged groove. A cluster of conserved residues comprises the active site of Ec-GyrA based on the crystal structure of the $59 \mathrm{kDa}$ fragment. Those residues (Ec-GyrA Arg32, Lys42, Arg46, Arg47, Arg121, Tyr122) are perfectly conserved among Pf- and Tg- GyrAs, consistent with their role and confirming that the cleavagereunion domain likely functions in an identical manner to that of other gyrases.

Next, the "GyrA-box" and its vicinity were considered. The GyrA-box is a positively charged motif (Sequence
$\mathrm{Q}++\mathrm{GG}+\mathrm{G}$, where + is any positively charged residue [42]) found in blade 1 of the $\beta$-pinwheel of the GyrA CTD and is known to be necessary for DNA supercoiling and wrapping $[44,45]$. GyrA from species such as E. coli have a conserved proline at position 636 (Ec-GyrA numbering) close to the hinge between blades 1 and 2 and this was thought to introduce a tilt in the packing between blades, causing the pinwheel to be out of plane, giving positive handedness to the DNA wrap [46]. Deletion of this proline in Ec-GyrA leads to a 2-3-fold decrease in supercoiling activity [46]. In some other species lacking the proline, such as B. burdorferi, the CTD is planar [17]. It is also planar in the case of 6-bladed Topo IV CTDs [47]. However, in $M$. tuberculosis gyrase, there is no conserved proline and yet the CTD is tilted [48]. These results suggest that other mechanisms in addition to/instead of the "conserved" proline are responsible for the non-planar shape.

Our alignments (Figure 2, Additional file 1: Figure S2) show that Pf-GyrA lacks a GyrA-box while a potential GyrA-box is present in Tg-GyrA (as a 996-RRGALGV1006 motif similar to the canonical sequence found in $E$. coli). It is notable that this Tg-GyrA box does not coincide with blade sequences as predicted by Pfam (Additional file 1: Figure S2) although it does coincide with the location of a $\beta$-pinwheel as predicted by I-TASSER when the sequence of Tg-GyrA CTD is submitted (see below). Interestingly, the sequence in place of the GyrA-box in Pf-GyrA has a negative charge (Pf-GyrA Asn821-Ser913 pI: 5.50). This stands out among other Plasmodium sequences in this region, which have pIs ranging between 7.19 and 8.17. The loop region containing the GyrA-box in Ec-GyrA (Ser555-Asp576) has a high pI of 8.16. The corresponding region of Tg-GyrA (Ser990-Ala1039) is similarly positively charged with its $\mathrm{pI}$ at 8.69 , which is in contrast to that of Pf-GyrA (Asn821-Tyr914, pI: 5.38). PfGyrA and Tg-GyrA both have apicoplast specific insertions in the region C-terminal to the GyrA-box (71 and 30 additional residues compared to Ec-GyrA, respectively). The moderately conserved proline (Ec-GyrA Pro636), is not present in either Plasmodium nor Toxoplasma gyrases. As a consequence of the lack of the GyrA box in Pf-gyrase we may expect that it is unable to efficiently supercoil DNA (as has been found for other gyrases lacking a GyrA box [44]). In the case of Tg-GyrA, four $\beta$ pinwheel blades predicted by Pfam are spread throughout the C-terminal region rather than being clustered together. In contrast, the homology model based on the mature amino acid sequence of Tg-GyrA by I-TASSER suggests that the majority of the $\beta$-pinwheel blades are located $\mathrm{N}$-terminal to those predicted by Pfam.

\section{Sequence analyses of GyrB}

The overall percentage of identical residues between EcGyrB and Pf-GyrB was 38.5\%. Unlike the case of Ec-GyrA 


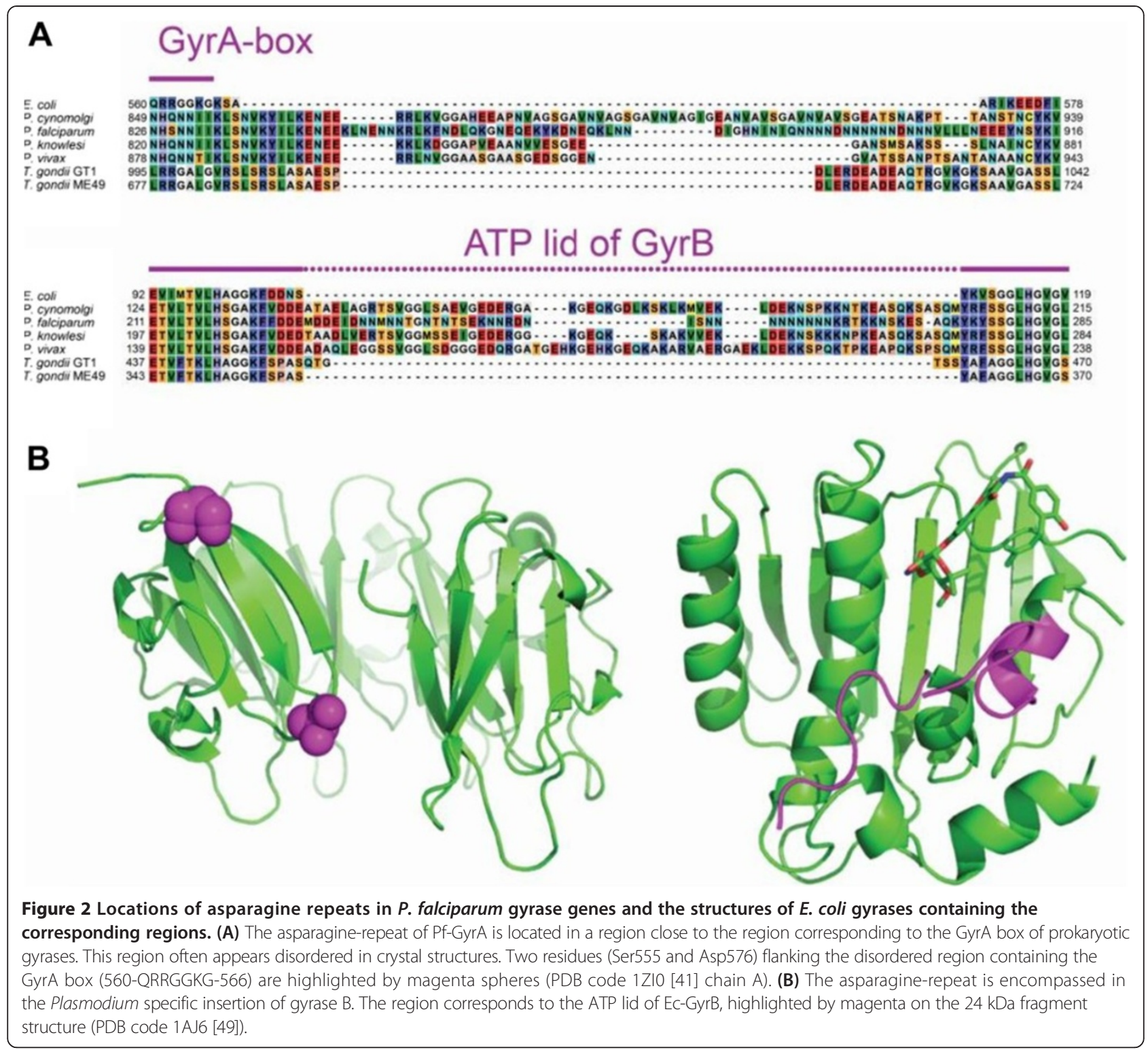

and Pf-GyrA, the identity is spread more homogenously throughout the length of the proteins. The percentage of identical residues at the $\mathrm{N}$-terminal domain $(E$. coli $31-$ 391 ) is $35.7 \%$, whereas for the C-terminal domain (E. coli $419-793)$ it is $40.7 \%$. The percentage of identical residues between Ec-GyrB and Tg-GyrB is 35.1\% (N-terminal domain: 35.6\%, C-terminal domain: 32.9\%).

Interestingly, Pf-GyrB includes an insertion of 45 amino acids in its TOPRIM domain which is essential for activity [37]. This same insert is present in all Plasmodium species but is absent from the other 9 GyrB genes we considered. Our alignments also confirmed a 49-amino acid insert in part of the ATPase domain corresponding to the ATP lid of Ec-GyrB (Figure 2). We find that this insert, which has been noted previously [34] is unique to Plasmodium GyrBs amongst the proteins we investigated.

\section{Size comparison}

The most striking difference between the proteins in comparison to their E. coli counterparts is in overall size. GyrA and GyrB in E. coli are 875 and 804 amino acids in length respectively; in Pf-gyrase the lengths of mature proteins are 1067 and 886 amino acids respectively while in $\mathrm{Tg}$ gyrase they are 1336 and 1041 amino acids respectively. The "extra" sequences in the apicomplexan gyrases are not entirely devoid of secondary structure as predicted by SOPMA [21] (Figure 1A, Figure 3). Those additional structural elements could confer unique properties to apicoplast gyrases, for example a 45 amino acid insertion in the TOPRIM domain of Pf-GyrB known to play a role in ATPase activity, DNA binding, and DNA cleavage [37]. There are cases, however, where the nonhomologous regions are predicted to form low complexity 


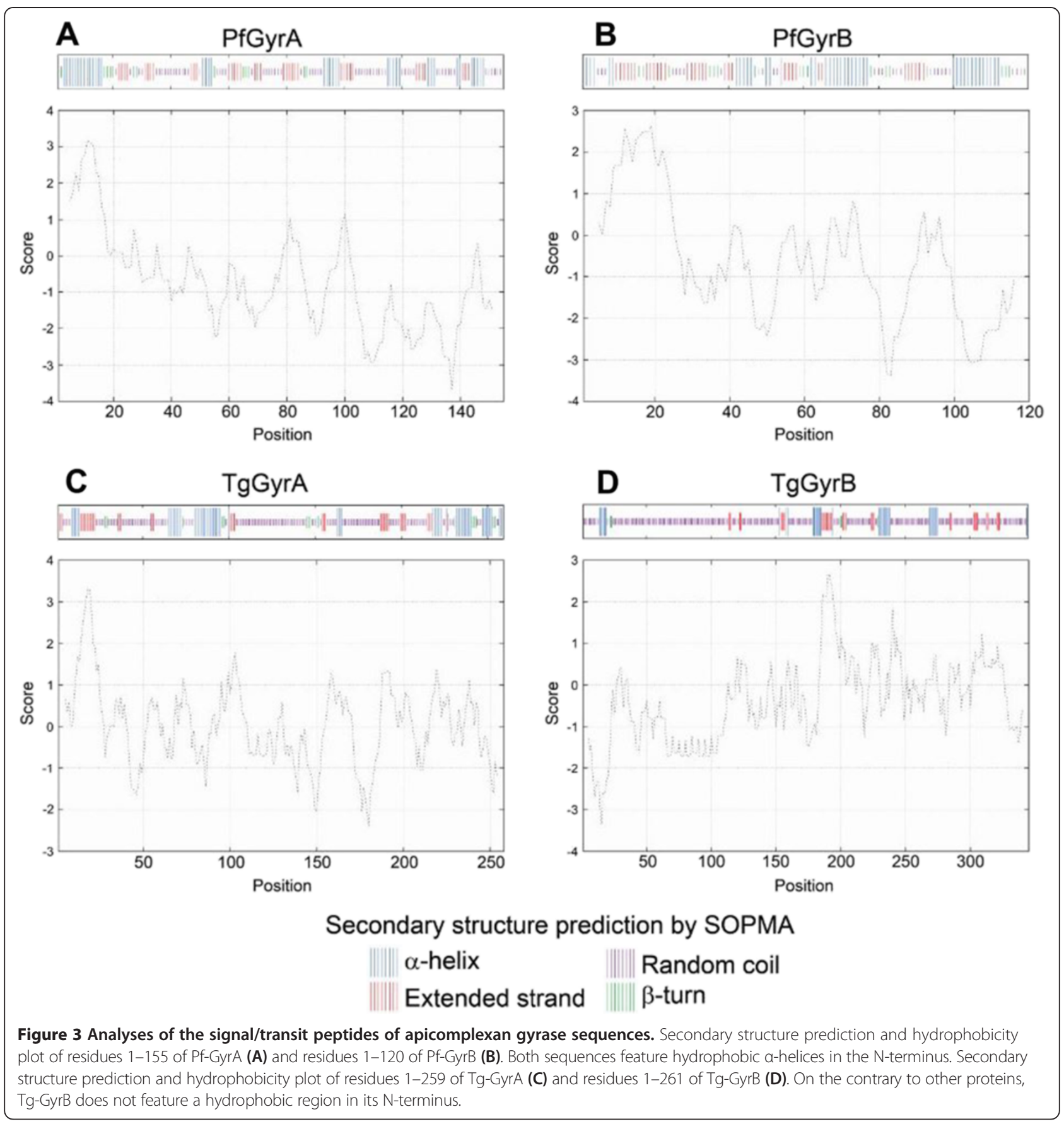

regions (LCRs), which appear correlated with single residue repeats. The $P$. falciparum proteome has previously been reported to be rich in asparagine repeats [50,51]. The feature is partially attributed to its AT-rich genome, but asparagine repeats are not found in other species of equally AT-rich Plasmodium spp. [51]. Asparagine repeats are thought to render the proteome susceptible to forming intracellular aggregates, but given the presence of a potent Hsp110 and other chaperones, they may provide a positive selective pressure by playing a role in immune evasion
$[52,53]$. The proportion of asparagine residues in the coding sequences of gyrase A and B were compared between four species in the Plasmodium genus: P. falciparum, $P$. vivax, $P$. cinomolgi, and $P$. knowlesi (Table 3). It is clear that the gyrase genes of $P$. falciparum encode the highest proportion of asparagine in accordance with Muralidharan and Goldberg [51]. Additionally, not only do Pf-gyrase proteins feature a quantitatively higher proportion of asparagine residues, but they are qualitatively unique because Pf-GyrA and Pf-GyrB each have regions where 
Table 3 Proportions of asparagine residues in the coding sequences of gyrases $A$ and $B$ in four species of Plasmodium

\begin{tabular}{lllll}
\hline & P. falciparum & P. vivax & P. cynomolgi & P. knowlesi \\
\hline Gyrase A & $14.65(10.97)$ & 7.44 & 7.98 & 9.04 \\
Gyrase B & $13.72(10.83)$ & 10.15 & 8.04 & 7.56 \\
\hline
\end{tabular}

Figures are given in percentages (\%). The figures in parentheses for $P$. falciparum are proportions of asparagine residues in the signal peptides (Pf-GyrA: 1-155, Pf-GyrB: 1-120).

asparagines are clustered into repeats (Additional file 1: Figure S3). For Pf-GyrB this region (253-261) is located in the area corresponding to the ATP-lid of the GHKL Bergerat fold of the ATPase domain [54], whereas in Pf-GyrA the repeat (888-902) is located in the region that corresponds to the GyrA box of the C-terminal $33 \mathrm{kDa}$ fragments in prokaryotic GyrA. In both cases the repeats are located in areas corresponding to loop regions in the predicted structures (Figure 2).

In contrast to Pf-gyrases, Tg-gyrases are rich in serines (Table 4). Unlike the asparagine repeats in Pf-gyrases, these serine repeats are primarily concentrated in the $\mathrm{N}$-terminal signal peptide regions (Additional file 1: Figure S4). Additionally, Tg-GyrB has a serine repeat in a region, which equates to the transducer domain of $E$. coli GyrB 43 kDa fragment.

We also compared the signal and transit peptides of the proteins. A typical apicoplast-targeting protein contains an N-terminal signal peptide followed by the apicoplast transit peptide for protein import into the apicoplast [55]. The amino acid sequence varies in transit peptides from different apicomplexans, for example, lysine is common in $P$. falciparum while arginine is common in $T$. gondii $[56,57]$. Those basic residues near the N-terminus of the transit peptide are reported to be important for faithful transport into the apicoplast and an algorithm has been established to identify the signal and transit peptides in multiple Apicomplexa species [58]. We analyzed Nterminal regions of Plasmodium and Toxoplasma gyrases that are expected to play key roles in translocation. Submission of Plasmodium and Toxoplasma gyrase sequences to the SOPMA [21] and Protscale [59] servers results in identification of a short hydrophobic $\alpha$-helix predicted for each Plasmodium gyrase gene (Figure 3).

Table 4 Proportion of serine resides in gyrases of $T$. gondii GT1 with respect to the full-length sequence and the signal peptide

\begin{tabular}{lll}
\hline & Full length protein & Signal peptide \\
\hline GyrA & $1-1594,9.91 \%$ & $1-258,23.26 \%$ \\
GyrB & $1-1386,20.06 \%$ & $1-345,40.58 \%$ \\
\hline
\end{tabular}

Signal peptide of T. gondii GT1 gyrases were inferred from aligned sequences (Additional file 1: Figure S5). Residue numbers are shown, followed by the proportion of serine residues as apercentage.
This is consistent with previous work showing that the signal peptides of apicoplast-targeted proteins in P. falciparum comprise a hydrophobic region [60]. In contrast, analyses of the Tg-GyrB sequence indicate different characteristics at the N-terminal region (Figure 3): Surprisingly for a protein that is predicted to be translocated to the apicoplast, the $\mathrm{N}$-terminal signal peptide of $\mathrm{Tg}$-GyrB is markedly non-hydrophobic which is in contrast to TgGyrA, Pf-GyrA and Pf-GyrB. This observation could imply an inefficient direction of $\mathrm{Tg}-\mathrm{GyrB}$ across the endoplasmic reticulum in the first step of the secretory pathway.

\section{Structure prediction and alignments \\ GyrA}

Homology modelling of full-length Pf-GyrA (minus the signal and transit peptide sequences) was carried out using I-TASSER $[61,62]$. The protein was predicted to align well with a number of known GyrA/ParC structures (see Additional file 1: Table S3) with the structure of full-length ParC from E. coli (1ZVU [42]) ranking top. Figure 4A shows the alignment between the predicted Pf-GyrA structure and ParC. The rmsd was $1.37 \AA$. The alignment shows that the N-terminus of Pf-GyrA approximately equivalent to the $59 \mathrm{kDa}$ region of $E$. coli GyrA aligns well with E. coli ParC, including the DNA breakage-reunion site, the coiled-coil region and the $\mathrm{C}$ gate.

Pf-GyrA CTD structure prediction by I-TASSER differs from Pfam results in that residues identified by Pfam as part of the $\beta$-pinwheels in the CTD (residues 978-1121, shown in orange in Figure 4A) do not align with the equivalent structure in ParC but residues 710968 do align with the 5-bladed ParC pinwheel. Given that the two predicted regions do not overlap, it may be that the actual structure consists of a large $\beta$-pinwheel consisting of both regions combined resulting in eight blades. This would be unusual as all gyrase $\beta$-pinwheels known have six blades. The equivalent region of topo IV in various organisms is more variable and includes both 3-bladed and 8-bladed pinwheels [42].

This inconsistency is attributable to a constraint to the structural homology calculations that derives from the fact that the ParC template structure features the pinwheel domain immediately $\mathrm{C}$-terminal to the $\mathrm{N}$-terminal domain. In order to create a homology model of the $\mathrm{C}$ terminal domain of Pf-GyrA without such constraint, the sequence of the C-terminal region (671-1222) was submitted to I-TASSER on its own. This results in the pinwheel domain being predicted in a region different from when the fuller sequence is submitted (Figure 4B). Homology models based on the five best templates consistently features the $\beta$-pinwheel domains beginning in residues $875-877$ and ending in residues $1220-1222$ 


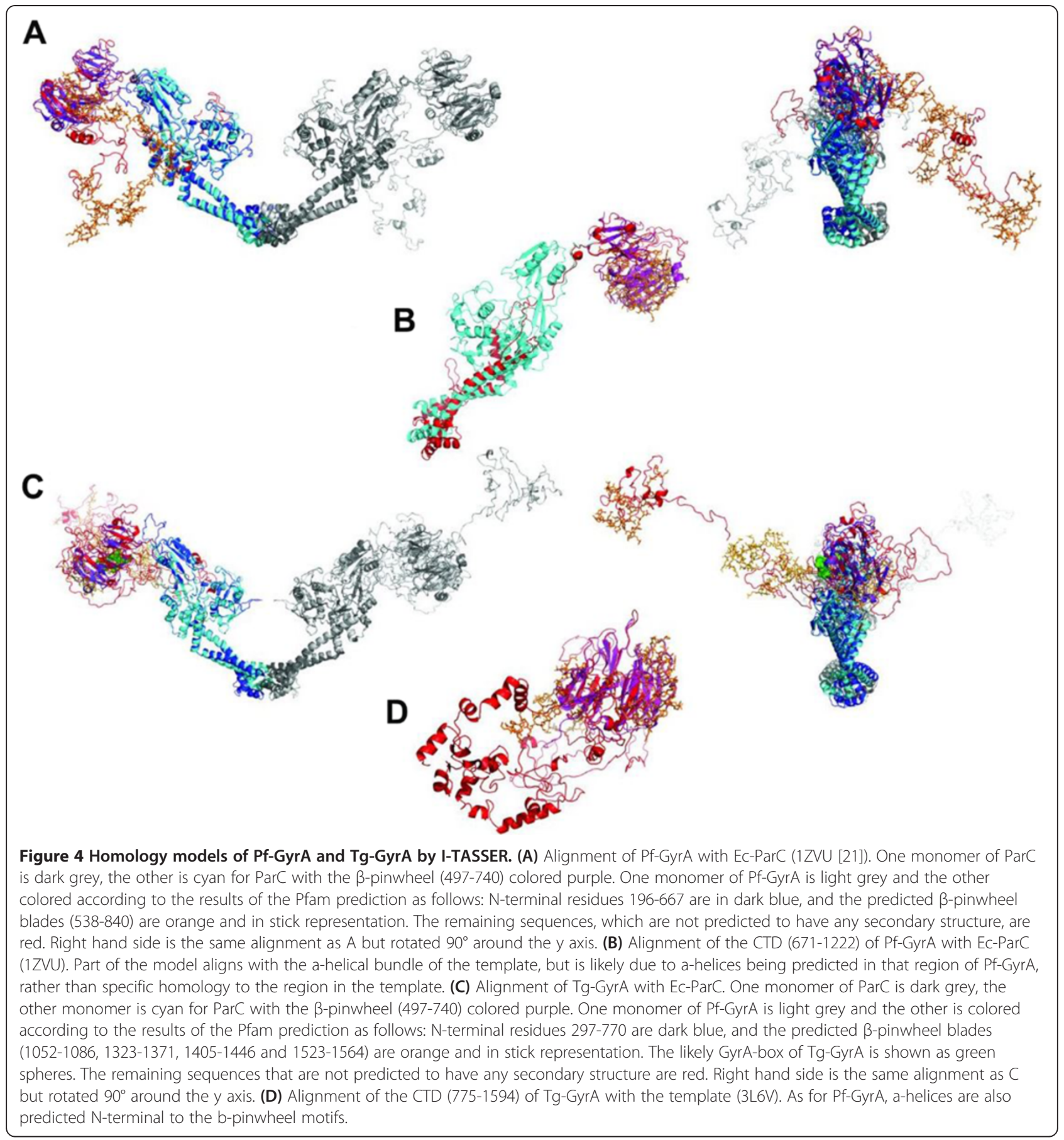

which covers all blades predicted by Pfam, and some blades predicted by I-TASSER using the mature sequence, to give a total of 6 blades. Also, the five best models consistently feature $\alpha$-helices between residues $698-848$. This is qualitatively similar to the prediction made by Dar et al. [35] in predicting a coiled-coil between the NTD and the CTD of GyrA, and hence legitimises the prediction of an additional structural element in Plasmodium GyrA compared to bacterial counterparts.

When the full length Tg-GyrA sequence (omitting the signal and transit sequences) was submitted to I-TASSER, the results as shown in Figure 4C were obtained. As with Pf-GyrA, the protein aligned best with the same E. coli ParC structure (pdb 1ZVU [42]). The rmsd was $1.91 \AA$. 
As was the case for the Pf-GyrA protein, there is good alignment with the cleavage reunion and $\mathrm{N}$-terminal portion of ParC. In addition, of the residues identified as $\beta$-pinwheel blades by Pfam; (1052-1086, 1323-1371, $1405-1446$ and 1523-1564) only the most N-terminal (1052-1086) overlaps with the $\beta$-pinwheel blades of $\operatorname{ParC}$, the others being dispersed throughout the Cterminus in regions not aligned with any part of the ParC structure. Interestingly, the region predicted to be the most $\mathrm{N}$-terminal blade is not recognized as any domain/motif by Pfam. The I-TASSER alignment places unaligned, largely unstructured sequences both $\mathrm{N}$ and $\mathrm{C}$-terminal to the $\beta$-pinwheel. The $\mathrm{C}$-terminal 500 residues beginning at residue A1096 of Tg-GyrA were not assigned as corresponding to any existing structure in ParC by I-TASSER. Similarly to Pf-GyrA, the sequence of the C-terminal region of Tg-GyrA (775-1594) was submitted to I-TASSER on its own (Figure 4D). The models based on the top four templates consistently predict $\alpha$-helices in residue range of 788-945, followed by $\beta$-pinwheel motifs between 974-1505. The latter range only covers three out of four blades predicted by Pfam but, as for the CTD of Pf-GyrA, a total of $6 \beta$ pinwheel blades is predicted in total. Clearly, the details of precisely which parts of the C-terminus are predicted to be a pinwheel depends on the software employed and the submitted amino acid sequence. Prediction of $\alpha$-helices $\mathrm{N}$-terminal to the $\beta$-pinwheel domain is similar to the case in Pf-GyrA, and suggests the presence of $\alpha$-helices between the NTD and the CTD as being a common element among apicomplexan GyrAs.

Homology models obtained from I-TASSER reveal little evidence of gross structural deviation by Pf-GyrA and $\mathrm{Tg}$-GyrA at the catalytic core and its surroundings. This is unsurprising given that the sequence identity is higher for the N-terminal domains in both GyrA and GyrB as described above. The most effective gyrase targeting antibacterials, the fluoroquinolone gyrase poisons, bind to a site made primarily of residues of the cleavage-reunion domains in GyrA and bound DNA [15]. The absolute conservation of amino acid sequence of this region in $\mathrm{Pf}$ and Tg-GyrA means that the binding site for gyrase poisons is likely unchanged. In Tg-GyrA, the exact start residue of the mature protein is not fully established, thus the possibility of a relatively large structural element at the $\mathrm{N}$-terminus is not completely ruled out.

\section{GyrB}

GyrB from $P$. falciparum lacking the signal and transit peptides was submitted to I-TASSER. The structure of full-length bacterial GyrB is not known and the alignment returned was best matched to a $S$. pneumoniae topo IV structure 4I3H [63] consisting of full-length ParE (equivalent to GyrB) fused to a $55 \mathrm{kDa}$ region of
ParC (equivalent to GyrA). The rmsd was $1.63 \AA$. The results (Figure 5A) shows that there is good structural homology overall with only a small number of continuous non-homologous regions. One interesting example is a region in the vicinity of the unique 49 amino acid insert (Pf-GyrB 224-272) previously identified in the ATP lid [34] (Figure 5A). Previous alignments suggested the 49 amino acid insert to be flanked by regions with high homology to other known GyrB sequences and form an unstructured loop [34]. On the contrary, the homology modelling by I-TASSER predicts the residues 225-320 to form a loop region (highlighted in Figure 5A). This inconsistency is likely an instance of "loop swapping" (see Methods section).

Tg-GyrB was also submitted to I-TASSER and like PfGyrB aligns well with the same S. pneumoniae topo IV structure $4 \mathrm{I} 3 \mathrm{H}$ [63] with an rmsd of $1.14 \AA$. Due to its extra length, Tg-GyrB contains a greater number of longer, unassigned loops. These loop regions are clustered around the GHKL region (Figure 5B). Overall, in both Pf- and Tg-GyrBs core domains seem to be largely preserved and this includes the regions involved in DNA binding (Figure 5).

GyrB contains the ATPase domain and it is clear from the aligned sequences of these domains that many key motifs are conserved between bacterial and apicoplast gyrases (Figure 6). Motifs conserved among GHKL fold termed N (uubEuuaNouDa), G1 (uxuxDNGxGuxbaauxxuu), G2 (uGxxGxouxSxxxuoxbuTuxT), and G3-box $\left(\mathrm{Tx}_{\mathrm{n}} \mathrm{GT}\right)$ can be identified ( $\mathrm{u}$, conserved bulky hydrophobic residues; $b$, basic residues; a, acidic residues) [54]. Indeed the ATPase activities of Pf-GyrB have been demonstrated $[35,37,64]$. The sequence of the GyrB subunit especially the ATP binding pocket is highly conserved among bacterial and apicoplast gyrases. For example, the residues located in a structural pocket, including N46, E50, R76, I78, K103, V118, and T165 known for ATP binding and ATPase inhibitors binding in EcGyrB [65] are conserved in Pf-GyrB. Two residues involved in the quinolone-binding pocket (also called quinolone resistance-determining region, QRDR $[66,67]$ ), N426 and K447 of Ec-GyrB are also conserved in Pf-GyrB. The similarity in drug interacting residues may explain the observation that known gyrase-targeting antibacterials drugs can show similar effects toward Pf-gyrase in ATPase activity and DNA cleavage activity assays [34,35]. A significant divergence is seen in Pf-GyrB where an insertion is present in the "lid" region covering the ATP binding site [34] (see below). In Tg-GyrB the clustering of continuous non-assigned sequences around the GHKL domain hints that this region is most structurally divergent and may even point to the presence of an additional domain. ATP binding and/or hydrolysis may also be affected in Pf-GyrB due to the insert in the ATP 

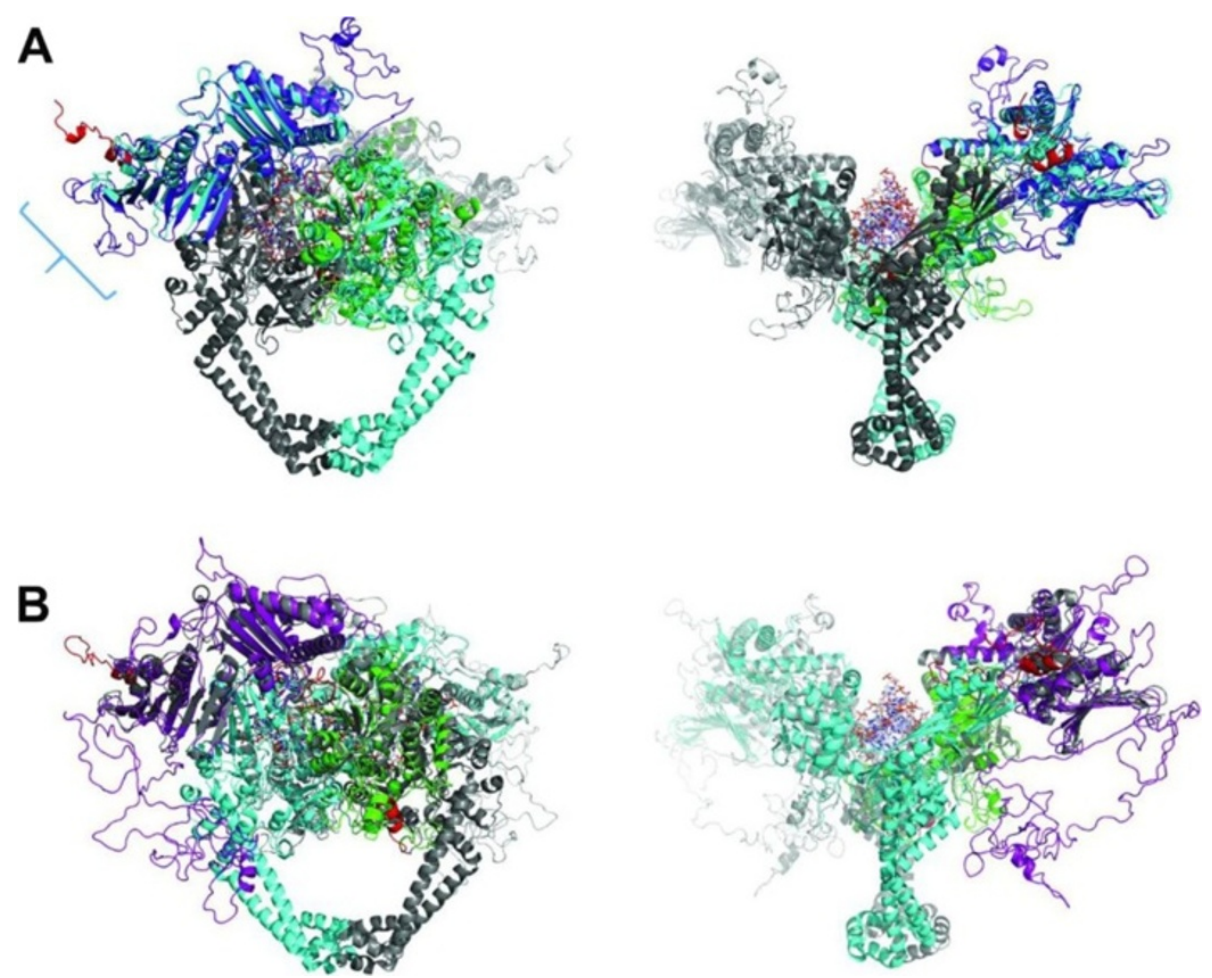

Figure 5 Homology models of Pf-GyrB and Tg-GyrB by I-TASSER. (A) Alignment of Pf-GyrB with topoisomerase IV complexed with DNA (pdb $4 \mathrm{I3YH}$ [63]). One monomer of the topo IV dimer is shown in dark grey, the other in cyan. One monomer of the Pf-GyrB is colored light grey, the other is colored as follows (numbering includes the signal and translocation peptide) residues 148-340 (roughly equivalent to GHKL domain), purple, residues 704-875 (roughly equivalent to the transducer domain, green) residues 910-1195 (roughly equivalent to TOPRIM and C-terminal tail), yellow. Unassigned sequence is shown in red. DNA bound to the DNA cleavage region is shown in stick format. Other DNA is removed for clarity. Bracketed loop is a Plasmodium specific insert. (B) Alignment of Tg-GyrB with Topoisomerase IV complex with DNA (pdb 4I3YH [63]). Coloring is as follows: Residues 291-876 (roughly equivalent to GHKL and transducer domains), purple, residues 910-1258 (roughly equivalent to TOPRIM and C-terminal tail), yellow. Unassigned sequence is shown in red. DNA bound to the DNA cleavage region is shown in stick format. Other DNA is removed for clarity. For each presentation, an alternative view rotated horizontally by $90^{\circ}$ is shown on right hand side.

lid, possibly explaining the decrease in hydrolysis rate compared to Ec-GyrB [35]. Indeed it has previously been speculated that the presence of unstructured loop regions in the ATPase domain may affect ATPase activity and the effectiveness of inhibitors, which bind in that region [34]. The extra sequence in this region could perhaps also provide scope for binding of new inhibitors.

\section{Conclusions}

In summary, we have carried out a comprehensive sequence and structural comparison of two apicomplexan

\begin{tabular}{|c|c|c|c|c|}
\hline & N-box & G1-box & P-loop & G3-box \\
\hline E. coli & $\begin{array}{l}\text { u u bEuuaNouDa } \\
\text { 39 MVFEVVDNAIDE } 50\end{array}$ & $\begin{array}{l}\text { u } \times \text { u } \times \text { XNG } \times \text { Gu } \times \text { ba au } \times \times \text { u u } \\
69 \text { VSVQDDGRGIPTGIHPEEG } 87\end{array}$ & $\begin{array}{c}\text { GXXGXG } \\
114 \text { GLHGVG } 119\end{array}$ & $\begin{array}{c}\text { GT } \\
161 \text { EKT GTMVR } 168\end{array}$ \\
\hline M. tuberculosis & 56 LIWEVVDNAVDE 67 & 86 VEVADDGRGIPVATHAS - G 103 & 130 GLHGVG 135 & 176 KKTGSTVR 183 \\
\hline S. aureus & 47 LVWE I VDNS I DE 58 & 77 IKVTDNGRG|PVDIQEKMG 95 & 122 GLHGVG 127 & 169 DKTGTVIR 176 \\
\hline S. enterica & 39 MVFEVVDNAIDE 50 & 69 VSVTDDGRG|PTG।HPEEG 87 & 114 GLHGVG 119 & 161 DKTGTMVR 168 \\
\hline X. campestris & 49 MVFEVVDNSVDE 60 & 79 VAVSDNGRGVPVDI HKEEG 97 & 124 GLHGVG 129 & 171 TKRGT TLR 178 \\
\hline E. coli ParC & 35 LGQEVIDNSVDE 46 & 65 LEVIDDGRGMPVDI HPEEG 83 & 110 GLHGVG 115 & 160 - NTGTSVH 166 \\
\hline P. cynomolgi & 71 I LFEI I DNSVDE 82 & 101 VTIEDNGRGIPCDIHEKTN 119 & 210 GLHGVG 215 & 258 SKRGTQIH 265 \\
\hline P. falciparum & 156 ILFEIIDNSVDE 167 & 186 VTIEDNGRGIPCDVHEKTK 204 & 280 GLHGVG 285 & 328 NKRGTQIH 335 \\
\hline P. knowlesi & 144 ILFEIIIDNSVDE 155 & 174 VTIEDNGRGIPCDIHEKTK 192 & 279 GLHGVG 284 & 327 SKRGTQIH 334 \\
\hline$P$. vivax & 86 ILFEIIIDNSVDE 97 & 116 VTIEDNGRG I PCDVHEKTK 134 & 233 GLHGVG 238 & 281 SKRGTQIH 288 \\
\hline T. gondii GT1 & 383 LVWEAI DNAVDE 394 & 414 VTVADDGRG IPCDVHPQTG 432 & 465 GLHGVG 470 & 588 PSSGTCLT 595 \\
\hline T. gondii ME49 & 290 LVWEA I DNAVDE 301 & 320 VTVADDGRG।PCDVHPQTG 338 & 265 GLHGVG 370 & 488 PSSGTCLT 495 \\
\hline
\end{tabular}


gyrases to standard bacterial (E. coli) gyrase and have found a number of differences which may have both relevance to enzyme function and consequences in terms of effectiveness of therapeutics as well as providing useful data for future structural studies.

While the results presented in this work are not definitive there are sufficient data to enable some tentative speculation, which should be considered in the light of recent findings from $M$. tuberculosis: This organism also has a gyrase but lacks topo IV. M. tuberculosis gyrase has a greater decatenation ability relative to negative supercoiling ability compared to Ec-gyrase [68,69], something which is thought to be due to decreased DNA-stimulated ATPase activity and a truncated "tail" in the CTD which combine to inhibit the enzyme from achieving as high a level of DNA duplex under-winding as the E. coli enzyme [48]. Further research has shown that this gyrase has a calcium binding site and that that gyrase in $M$. tuberculosis can shift between gyrase-like and topo IV-like activities via the modulatory action of $\mathrm{Ca}^{2+}$ which binds in the linker region between the NTD and CTD of GyrA [70]. This is proposed to alter the position of the $\beta$-pinwheels relative to the remainder of the enzyme such that supercoiling may be favored in the absence of $\mathrm{Ca}^{2+}$ while disfavored in its presence, allowing relaxation/decantation to occur. The fact that the greatest variation in Pf- and Tg-gyrases compared to Ec-gyrase is in the CTD strongly suggests that this is due to a role in affecting or modulating the balance between gyrase-like and topo IV-like activities. Tggyrase, in contrast to Pf-gyrase, has a likely GyrA box but lacks an insert in the ATP lid meaning that these mooted activity modifiers are not available to it. In this instance, compensation for the lack of topo IV is presumably achieved at least in part through its unusually large GyrA CTD, which may affect extent and/or position of the DNA wrap. It is also the case that additional structural elements found in Plasmodium and Toxoplasma gyrases may be responsible for modulating their activity by means not found in prokaryotes. These may pose an additional target for drugs in the future. For example, we may envisage that inhibiting the apicoplast targeting of gyrases will be lethal to Apicomplexa.

Further biochemical studies on individual gyrase proteins from these organisms as well as holoenzymes in conjunction with high-resolution studies will be required to answer outstanding questions and assess their suitability as targets for development of therapeutics.

\section{Methods}

\section{Sequence comparisons}

Amino acid sequences of proteins were aligned with ClustalW 2.1 using the default parameters [39]. The percentages of sequence identities were calculated by dividing the number of identical residues by the total aligned sequence length without insertions. Aligned sequences were viewed using CLC Sequence Viewer (CLC bio). Aligned amino acid sequences of gyrase and topoisomerase IV proteins from various species were inputted into MEGA (v. 6.06 beta) [38]. Pairwise distances between sequences were computed (the number of amino acid differences divided by the total number of amino acids compared). Gaps and missing data were deleted and no variance estimation method was used. A maximum parsimony tree was calculated using the JonesTaylor-Thornton model assuming uniform rates among sites. Phylogeny was tested using the bootstrap method with 500 bootstrap replications.

\section{Structure prediction}

Structure predictions were carried out using I-TASSER $[61,62]$. In all cases, amino acid sequences for the proteins, lacking the predicted signal and transit sequences were submitted to the I-TASSER server. Default settings were used with no additional restraints employed. From the top 10 identified structural analogues returned the best alignment was chosen based on TM-score ranking. The top ranking alignment templates were E. coli ParC [63] for Pf-GyrA, PfGyrA CTD (residues 671-1222) and Tg-GyrA, S. pneumoniae ParC-ParE55 fusion protein [63] for Pf-GyrB and TgGyrB and X. campestris GyrA CTD for Tg-GyrA CTD (residues 775-1594). PDB coordinates were visualised using PyMOL [71]. All of the proteins considered were significantly longer than the homologous regions in the model templates. This has an important consequence for the homology models generated. When additional sequences are present then clearly not all sequences can be aligned to a template. In some cases we observe that where sequences flanking these loop regions themselves have poor similarity to the template, then "loop swapping" may occur where the flanking sequence is in fact designated a loop and the loop is assigned structure. This does occur in parts of our structure but has little effect on the overall model.

\section{Structural alignments}

Dimeric models of homology models of the proteins as generated by I-TASSER, were created by superposing each homology model onto dimeric crystal structures of the template topoisomerases using Coot [72]. The SSM Superpose function was used, and root mean square deviation (RMSD) of the distances between alpha carbons of the aligned residues were calculated. This allowed us to see regions of the Pf or Tg protein, which matched well with a known gyrase protein structure and, more importantly those regions where poor homology was predicted.

\section{Availability of supporting data}

All supporting data associated with this manuscript are included as additional files. 


\section{Additional file}

\section{Additional file 1. Provides additional data as follows: Additional Tables 1 and 2: Estimates of evolutionary divergence between various GyrA sequences and between various GyrB sequences, Additional Figure 1: Showing molecular phylogenetic analyses for GyrA (and ParC) and GyrB (and ParE). Additional Figure 2: A figure showing sequence alignment of predicted $b$-pinwheel blades of GyrA. Additional Figure 3: A figure showing the location of asparagine residues in Pf-gyrase proteins. Additional Figure 4: A figure showing the location of serine residues in Tg-gyrase proteins. Additional Figure 5: Sequence alignments used to infer the starting position of the mature gyrase proteins of $T$. gondii GT1.}

\section{Competing interests}

The authors declare that they have no competing interests.

\section{Authors' contributions}

SN, TL and JR designed and carried out experiments, analysed results and wrote the paper. JGH designed, carried out experiments, oversaw the project, analysed results and wrote the paper. All authors read and approved the final manuscript.

\section{Acknowledgement}

The authors are grateful to Kam Zhang for critical reading of the manuscript.

\section{Funding}

This work was supported by RIKEN Initiative Research Funding awarded to $\mathrm{JGH}$.

\section{Author details}

${ }^{1}$ Heddle Initiative Research Unit, RIKEN, 2-1 Hirosawa, Wako, Saitama 351-0198, Japan. ${ }^{2}$ Current address: Department of Molecular Protozoology, Research Institute for Microbial Diseases (RIMD), Osaka University, 3-1 Yamadaoka, Suita, Osaka 565-0871, Japan.

Received: 24 July 2014 Accepted: 10 December 2014

Published online: 19 December 2014

\section{References}

1. Lim L, McFadden Gl: The evolution, metabolism and functions of the apicoplast. Phil Trans R Soc B 2010, 365(1541):749-763.

2. van Dooren $G G$, Striepen $B$ : The algal past and parasite present of the apicoplast. Annu Rev Microbio/ 2013, 67(1):null.

3. WHO: World Malaria Report. Geneva: World Health Organization Press; 2012.

4. Centers for Disease Control and Prevention [http://www.cdc.gov/parasites/ npi.html]

5. Petersen I, Eastman R, Lanzer M: Drug-resistant malaria: molecular mechanisms and implications for public health. FEBS Lett 2011, 585(11):1551-1562.

6. Dondorp AM, Nosten F, Yi P, Das D, Phyo AP, Tarning J, Lwin KM, Ariey F, Hanpithakpong W, Lee SJ, Ringwald P, Silamut K, Imwong M, Chotivanich K, Lim P, Herdman T, An SS, Yeung S, Singhasivanon P, Day NP, Lindegardh N, Socheat $D$, White $N J$ : Artemisinin resistance in Plasmodium falciparum malaria. New Engl J Med 2009, 361(5):455-467.

7. Anderson TJ, Nair S, Nkhoma S, Williams JT, Imwong M, Yi P, Socheat D, Das D, Chotivanich K, Day NP, White NJ, Dondorp AM: High heritability of malaria parasite clearance rate indicates a genetic basis for artemisinin resistance in western Cambodia. J Infect Dis 2010, 201(9):1326-1330.

8. The RTS, S Clinical Trials Partnership.: A phase 3 trial of RTS,S/AS01 malaria vaccine in African infants. New Engl J Med 2012, 367(24):2284-2295.

9. Seder RA, Chang L-J, Enama ME, Zephir KL, Sarwar UN, Gordon IJ, Holman LA, James ER, Billingsley PF, Gunasekera A, Richman A, Chakravarty S, Manoj A, Velmurugan S, Li ML, Ruben AJ, Li T, Eappen AG, Stafford RE, Plummer SH, Hendel CS, Novik L, Costner PJM, Mendoza FH, Saunders JG, Nason MC, Richardson JH, Murphy J, Davidson SA, Richie TL, et al: Protection against malaria by intravenous immunization with a nonreplicating sporozoite vaccine. Science 2013. 341:1359-1365

10. Sims PG: Drug Resistance in Toxoplasma gondii. In: Antimicrobial Drug Resistance. Edited by Mayers D: Humana Press; New York: 2009: 1121-1126.
11. Fichera ME, Roos DS: A plastid organelle as a drug target in apicomplexan parasites. Nature 1997, 390(6658):407-409.

12. He CY, Shaw MK, Pletcher CH, Striepen B, Tilney LG, Roos DS: A plastid segregation defect in the protozoan parasite Toxoplasma gondii. Embo $J$ 2001, 20(3):330-339.

13. Yeh $\mathrm{E}$, DeRisi J L: Chemical rescue of malaria parasites lacking an apicoplast defines organelle function in blood-stage Plasmodium falciparum. PLOS Biol 2011, 9(8):e1001138.

14. Pradel G, Schlitzer M: Antibiotics in malaria therapy and their effect on the parasite apicoplast. Curr Mol Med 2010, 10(3):335-349.

15. Collin F, Karkare S, Maxwell A: Exploiting bacterial DNA gyrase as a drug target: current state and perspectives. Appl Microbiol Biotechnol 2011, 92(3):479-497

16. Nöllmann M, Crisona NJ, Arimondo PB: Thirty years of escherichia coli DNA gyrase: from in vivo function to single-molecule mechanism. Biochimie 2007, 89(4):490-499.

17. Corbett KD, Shultzaberger RK, Berger JM: The C-terminal domain of DNA gyrase A adopts a DNA-bending B-pinwheel fold. Proc Natl Acad Sci U S A 2004, 101(19):7293-7298.

18. Chaudhuri I, Soding J, Lupas AN: Evolution of the beta-propeller fold. Proteins 2008, 71(2):795-803.

19. Aravind L, Leipe DD, Koonin EV: Toprim - a conserved catalytic domain in type IA and II topoisomerases, DnaG-type primases, OLD family nucleases and RecR proteins. Nucl Acids Res 1998, 26(18):4205-4213.

20. Punta M, Coggill PC, Eberhardt RY, Mistry J, Tate J, Boursnell C, Pang N, Forslund K, Ceric G, Clements J, Heger A, Holm L, Sonnhammer EL, Eddy SR, Bateman A, Finn RD: The Pfam protein families database. Nucl Acids Res 2012, 40(Database issue):D290-D301.

21. Geourjon C, Deleage G: SOPMA: significant improvements in protein secondary structure prediction by consensus prediction from multiple alignments. Comput Appl Biosci 1995, 11(6):681-684.

22. Schoeffler AJ, Berger JM: Recent advances in understanding structurefunction relationships in the type II topoisomerase mechanism. Biochem Soc Trans 2005, 33(Pt 6):1465-1470.

23. Wall MK, Mitchenall LA, Maxwell A: Arabidopsis thaliana DNA gyrase is targeted to chloroplasts and mitochondria. Proc Natl Acad Sci USA 2004, 101(20):7821-7826.

24. Divo AA, Sartorelli AC, Patton CL, Bia FJ: Activity of fluoroquinolone antibiotics against Plasmodium falciparum in vitro. Antimicrob Agents Chemother 1988, 32(8):1182-1186.

25. Weissig V, Vetro-Widenhouse TS, Rowe TC: Topoisomerase II inhibitors induce cleavage of nuclear and 35-kb plastid DNAs in the malarial parasite Plasmodium falciparum. DNA Cell Biol 1997, 16(12):1483-1492.

26. Krishna S, Davis TM, Chan PC, Wells RA, Robson KJ: Ciprofloxacin and malaria. Lancet 1988, 1(8596):1231-1232.

27. Khan AA, Slifer T, Araujo FG, Remington JS: Trovafloxacin is active against Toxoplasma gondii. Antimicrob Agents Chemother 1996, 40(8):1855-1859.

28. Gardner MJ, Hall N, Fung E, White O, Berriman M, Hyman RW, Carlton JM, Pain A, Nelson KE, Bowman S, Paulsen IT, James K, Eisen JA, Rutherford K, Salzberg SL, Craig A, Kyes S, Chan MS, Nene V, Shallom SJ, Suh B, Peterson J, Angiuoli S, Pertea M, Allen J, Selengut J, Haft D, Mather MW, Vaidya AB, Martin DM, et al: Genome sequence of the human malaria parasite Plasmodium falciparum. Nature 2002, 419(6906):498-511.

29. García-Estrada C, Prada CF, Fernández-Rubio C: Rojo-Vázquez F, Balaña-Fouce R: DNA topoisomerases in apicomplexan parasites: promising targets for drug discovery. P Roy Soc B-Biol Sci 2010, 277(1689):1777-1787.

30. Gozalbes R, Brun-Pascaud M, Garcia-Domenech R, Galvez J, Girard PM, Doucet JP, Derouin F: Anti-toxoplasma activities of 24 quinolones and fluoroquinolones in vitro: prediction of activity by molecular topology and virtual computational techniques. Antimicrob Agents Chemother 2000, 44(10):2771-2776.

31. Mahmoudi N, Ciceron L, Franetich JF, Farhati K, Silvie O, Eling W, Sauerwein R, Danis M, Mazier D, Derouin F: In vitro activities of 25 quinolones and fluoroquinolones against liver and blood stage Plasmodium spp. Antimicrob Agents Chemother 2003, 47(8):2636-2639.

32. Piddock $\sqcup$, Zhu M: Mechanism of action of sparfloxacin against and mechanism of resistance in gram-negative and gram-positive bacteria. Antimicrob Agents Chemother 1991, 35(11):2423-2427.

33. Goodman CD, Su V, McFadden GI: The effects of anti-bacterials on the malaria parasite Plasmodium falciparum. Mol Biochem Parasitol 2007, 152(2):181-191. 
34. Raghu Ram EV, Kumar A, Biswas S, Chaubey S, Siddiqi MI, Habib S: Nuclear gyrB encodes a functional subunit of the Plasmodium falciparum gyrase that is involved in apicoplast DNA replication. Mol Biochem Parasitol 2007, 154(1):30-39.

35. Dar MA, Sharma A, Mondal N, Dhar SK: Molecular cloning of apicoplasttargeted plasmodium falciparum DNA gyrase genes: unique intrinsic ATPase activity and ATP-independent dimerization of PfGyrB subunit. Eukaryot Cell 2007, 6(3):398-412.

36. Ali JA, Jackson AP, Howells AJ, Maxwell A: The 43-kilodalton N-terminal fragment of the DNA gyrase B protein hydrolyzes ATP and binds coumarin drugs. Biochemistry 1993, 32(10):2717-2724.

37. Dar A, Prusty D, Mondal N, Dhar SK: A unique 45-amino-acid region in the toprim domain of plasmodium falciparum gyrase $B$ is essential for its activity. Eukaryot Cell 2009, 8(11):1759-1769.

38. Tamura K, Stecher G, Peterson D, Filipski A, Kumar S: MEGA6: Molecular Evolutionary Genetics Analysis version 6.0. Mol Biol Evol 2013, 30(12):2725-2729.

39. Larkin MA, Blackshields G, Brown NP, Chenna R, McGettigan PA, McWilliam $H$, Valentin F, Wallace IM, Wilm A, Lopez R, Thompson JD, Gibson TJ, Higgins DG: Clustal W and Clustal X version 2.0. Bioinformatics 2007, 23 (21):2947-2948.

40. Thompson JD, Higgins DG, Gibson TJ: CLUSTAL W: improving the sensitivity of progressive multiple sequence alignment through sequence weighting, position-specific gap penalties and weight matrix choice. Nucl Acids Res 1994, 22(22):4673-4680

41. Ruthenburg AJ, Graybosch DM, Huetsch JC, Verdine GL: A superhelical spiral in the Escherichia coli DNA gyrase A C-terminal domain imparts unidirectional supercoiling bias. J Biol Chem 2005, 280(28):26177-26184.

42. Corbett KD, Schoeffler AJ, Thomsen ND, Berger JM: The structural basis for substrate specificity in DNA topoisomerase IV. J Mol Biol 2005, 351(3):545-561.

43. Morais Cabral JH, Jackson AP, Smith CV, Shikotra N, Maxwell A, Liddington RC: Crystal structure of the breakage-reunion domain of DNA gyrase. Nature 1997, 388(6645):903-906.

44. Kramlinger VM, Hiasa $\mathrm{H}$ : The "GyrA-box" is required for the ability of DNA gyrase to wrap DNA and catalyze the supercoiling reaction. J Biol Chem 2006, 281(6):3738-3742.

45. Ward D, Newton A: Requirement of topoisomerase IV parC and parE genes for cell cycle progression and developmental regulation in Caulobacter crescentus. Mol Microbiol 1997, 26(5):897-910.

46. Hsieh TJ, Yen TJ, Lin TS, Chang HT, Huang SY, Hsu CH, Farh L, Chan NL: Twisting of the DNA-binding surface by a beta-strand-bearing proline modulates DNA gyrase activity. Nucleic Acids Res 2010, 38(12):4173-4181.

47. Hsieh TJ, Farh L, Huang WM, Chan NL: Structure of the topoisomerase IV C-terminal domain: a broken beta-propeller implies a role as geometry facilitator in catalysis. J Biol Chem 2004, 279(53):55587-55593.

48. Tretter EM, Berger JM: Mechanisms for defining supercoiling Set point of DNA gyrase orthologs: II. The shape of the GyrA subunit C-terminal domain $(\mathrm{Ctd})$ is Not a sole determinant for controlling supercoiling efficiency. J Biol Chem 2012, 287(22):18645-18654

49. Holdgate GA, Tunnicliffe A, Ward WH, Weston SA, Rosenbrock G, Barth PT, Taylor IW, Pauptit RA, Timms D: The entropic penalty of ordered water accounts for weaker binding of the antibiotic novobiocin to a resistant mutant of DNA gyrase: a thermodynamic and crystallographic study. Biochemistry 1997, 36(32):9663-9673.

50. Aravind $L$, lyer $L M$, Wellems TE, Miller LH: Plasmodium biology: genomic gleanings. Cell 2003, 115(7):771-785.

51. Muralidharan $V$, Goldberg DE: Asparagine repeats in plasmodium falciparum proteins: good for nothing? PLoS Pathog 2013, 9(8):e1003488.

52. Verra F, Hughes AL: Biased amino acid composition in repeat regions of Plasmodium antigens. Mol Biol Evol 1999, 16(5):627-633.

53. Hughes AL: The evolution of amino acid repeat arrays in Plasmodium and other organisms. J Mol Evol 2004, 59(4):528-535.

54. Dutta R, Inouye M: GHKL, an emergent ATPase/kinase superfamily. Trends Biochem Sci 2000, 25(1):24-28.

55. Waller RF, Keeling PJ, Donald RG, Striepen B, Handman E, Lang-Unnasch N, Cowman AF, Besra GS, Roos DS, McFadden Gl: Nuclear-encoded proteins target to the plastid in Toxoplasma gondii and Plasmodium falciparum. Proc Natl Acad Sci U S A 1998, 95(21):12352-12357.

56. Parsons M, Karnataki A, Feagin JE, DeRocher A: Protein trafficking to the apicoplast: deciphering the apicomplexan solution to secondary endosymbiosis. Eukaryot Cell 2007, 6(7):1081-1088.
57. Ralph SA, Foth BJ, Hall N, McFadden Gl: Evolutionary pressures on apicoplast transit peptides. Mol Biol Evol 2004, 21(12):2183-2194.

58. Cilingir G, Broschat SL, Lau AO: ApicoAP: the first computational model for identifying apicoplast-targeted proteins in multiple species of Apicomplexa. PLOS ONE 2012, 7(5):e36598.

59. Gasteiger E, Hoogland C, Gattiker A, Duvaud Se, Wilkins M, Appel R, Bairoch A: Protein Identification and Analysis Tools on the ExPASy Server. In: The Proteomics Protocols Handbook. Edited by Walker J: Humana Press; New York: 2005: 571-607.

60. Foth BJ, Ralph SA, Tonkin CJ, Struck NS, Fraunholz M, Roos DS, Cowman AF, McFadden Gl: Dissecting apicoplast targeting in the malaria parasite Plasmodium falciparum. Science 2003, 299(5607):705-708.

61. Zhang Y: I-TASSER server for protein 3D structure prediction. BMC Bioinformatics 2008, 9(1):40.

62. Roy A, Kucukural A, Zhang Y: I-TASSER: a unified platform for automated protein structure and function prediction. Nat Protocols 2010, 5(4):725-738.

63. Laponogov I, Veselkov DA, Crevel IM-T, Pan X-S, Fisher LM, Sanderson MR: Structure of an 'open' clamp type II topoisomerase-DNA complex provides a mechanism for DNA capture and transport. Nucl Acids Res 2013, 41(21):9911-9923.

64. Khor V, Yowell C, Dame JB, Rowe TC: Expression and characterization of the ATP-binding domain of a malarial Plasmodium vivax gene homologous to the B-subunit of the bacterial topoisomerase DNA gyrase. Mol Biochem Parasitol 2005, 140(1):107-117.

65. Ostrov DA, Hernandez Prada JA, Corsino PE, Finton KA, Le N, Rowe TC: Discovery of novel DNA gyrase inhibitors by high-throughput virtual screening. Antimicrob Agents Chemother 2007, 51(10):3688-3698.

66. Yoshida H, Bogaki M, Nakamura M, Nakamura S: Quinolone resistancedetermining region in the DNA gyrase gyrA gene of Escherichia coli. Antimicrob Agents Chemother 1990, 34(6):1271-1272.

67. Yoshida H, Bogaki M, Nakamura M, Yamanaka LM, Nakamura S: Quinolone resistance-determining region in the DNA gyrase gyrB gene of Escherichia coli. Antimicrob Agents Chemother 1991, 35(8):1647-1650.

68. Aubry A, Fisher LM, Jarlier V, Cambau E: First functional characterization of a singly expressed bacterial type II topoisomerase: the enzyme from Mycobacterium tuberculosis. Biochem Biophys Res Comm 2006, 348(1):158-165.

69. Manjunatha UH, Dalal M, Chatterji M, Radha DR, Visweswariah SS, Nagaraja $\checkmark$ : Functional characterisation of mycobacterial DNA gyrase: an efficient decatenase. Nucleic Acids Res 2002, 30(10):2144-2153.

70. Karkare S, Yousafzai F, Mitchenall LA, Maxwell A: The role of $\mathrm{Ca} 2+$ in the activity of Mycobacterium tuberculosis DNA gyrase. Nucleic Acids Res 2012, 40(19):9774-9787.

71. Schrodinger, LLC: The PyMOL Molecular Graphics System, Version 1.3r1. In.; 2010.

72. Emsley P, Lohkamp B, Scott WG, Cowtan K: Features and development of Coot. Acta Crystallogr D 2010, 66(Pt 4):486-501

\section{Submit your next manuscript to BioMed Central and take full advantage of:}

- Convenient online submission

- Thorough peer review

- No space constraints or color figure charges

- Immediate publication on acceptance

- Inclusion in PubMed, CAS, Scopus and Google Scholar

- Research which is freely available for redistribution 